УДК 533.9.08

\title{
ACTIVATION NEUTRON DETECTOR FOR PULSE REACTION OF D-D SYNTHESIS
}

\author{
S.A. Dan'ko ${ }^{1}$, S.S. Kingsep ${ }^{1}$, O.V. Tel'kovskaya ${ }^{1}$, A.G. Alekseev ${ }^{2}$, G.I. Britvich ${ }^{2}$ \\ ${ }^{1}$ NRC "Kurchatov Institute", Moskow, Russia \\ ${ }^{2}$ Institute for High Energy Physics, Protvino, MR, Russia
}

This paper presents a detector of neutrons generated in nuclear fusion reaction in hot deuterium plasma during short time of $10^{-8} \mathrm{~s}$ of its existence. Operation principle of detection is based on the slowdown of the fast neutrons up to thermal energies and activation by them of indium nuclei that decay with generation of beta-particles with half-decay period of $14 \mathrm{~s}$ which is used in the device. Extended over time process of registration of beta particles avoids interferences associated with pulsed electromagnetic fields and $X$-rays that accompany the action of powerful current generators. Dynamic range of neutron detector exceeds $10^{5}$. Detector calibration was carried out, and numerical calculations of slowing down of fast neutron and activation of indium were made. The difference between neutron energy distribution of standard ${ }^{252} \mathrm{Cf}$ isotope and the energy of neutrons arising in experimental $\mathrm{D}-\mathrm{D}$ reaction is taken into account.

Key words: hot plasma, neutron detector, activation method, calibration, californium-252, numerical calculation.

\section{АКТИВАЦИОННЫЙ ДЕТЕКТОР НЕЙТРОНОВ ДЛЯ ИМПУЛЬСНОЙ РЕАКЦИИ D-D-CИНТЕЗА \\ С.А. Данько ${ }^{1}$, С.С. Кингсеп ${ }^{1}$, О.В. Тельковская ${ }^{1}$, А.Г. Алексеев ${ }^{2}$, Г.И. Бритвич² \\ ${ }^{1}$ НИЦ «Курчатовский институт», Москва, Россия \\ ${ }^{2}$ ГНЦ Институт физики высоких энергий, Протвино, МО, Россия}

В работе представлен детектор нейтронов, генерируемых в реакции ядерного синтеза в горячей дейтериевой плазме за короткое время $10^{-8}$ с её существования. Принцип действия детектора основан на замедлении быстрых нейтронов до тепловых энергий и активации ими индия, который, распадаясь, образует $\beta$-частицы с временем полураспада 14 с. Растянутый во времени процесс регистрации $\beta$-частиц позволяет избежать помех, связанных с импульсными электромагнитными полями и рентгеновским излучением, которые сопровождают работу мощных генераторов тока. Динамический диапазон детектора превышает $10^{5}$. Проведена градуировка детектора, и сделаны численные расчёты замедления быстрых нейтронов и активации ими индия. При градуировке учтена разница в энергиях нейтронов эталонного источника ${ }^{252} \mathrm{Cf}$ и нейтронов, получаемых в результате $\mathrm{D}-\mathrm{D}-$ синтеза, для регистрации которых предназначен детектор.

Ключевые слова: горячая плазма, детектор нейтронов, активационный метод, градуировка, калифорний-252, численные расчёты.

\section{ВВЕДЕНИЕ}

Реакция синтеза лёгких ядер в импульсных плазменных разрядах на мощных генераторах тока характеризуется короткой длительностью $\sim 10^{-8}$ с и пространственной локализацией $\sim 1 \mathrm{cm.} \mathrm{Обычно} \mathrm{в} \mathrm{каче-}$ стве тестовой используется реакция на ядрах дейтерия, в одной из двух ветвей которой генерируются нейтроны, беспрепятственно выходящие из зоны реакции и вследствие этого являющиеся удобным продуктом для диагностики высокотемпературной плазмы. Полный выход нейтронов в современных экспериментах составляет $10^{9}-10^{12}$ частиц.

Измерение нейтронных потоков обычно производится сцинтилляционными детекторами, в частности, по времени пролёта известного расстояния с высокой точностью можно определить энергию нейтронов. Но при таких коротких импульсах излучения (до 10 нс) возникают проблемы с динамическим диапазоном измерений у сцинтилляционных детекторов, что сильно ограничивает возможность их использования в нашем случае.

\section{ОПИСАНИЕ ДЕТЕКТОРА}

Для измерения полного нейтронного выхода в импульсной D-D-реакции в данной работе использовался детектор, который основан на активационной методике с затянутой регистрацией: сначала быстрые нейтроны замедляются в полиэтиленовом кубе, ребро которого имеет длину 9 см; далее уже замедленные нейтроны взаимодействуют с шестью индиевыми пластинами толщиной 1,5 мм и площадью $6,5 \times 8,5$ см, расположенными на гранях куба, с образованием $\beta$-активных ядер в реакции ${ }^{115} \operatorname{In}(n, \gamma){ }^{116} \mathrm{In}$. Для регистрации использовался канал с временем полураспада ${ }^{116}$ In $T_{1 / 2} \square=14,1$ с, существует ещё второй канал с временем полураспада 55 мин. Излучённые $\beta$-частицы регистрировались шестью счётчиками Гейгера (СБТ-10), приложенными к обратной стороне индиевых пластин. Вся сборка помещена в электростатический экран из алюминия, имеющий форму куба с ребром 250 мм. Фотография детектора представле- 


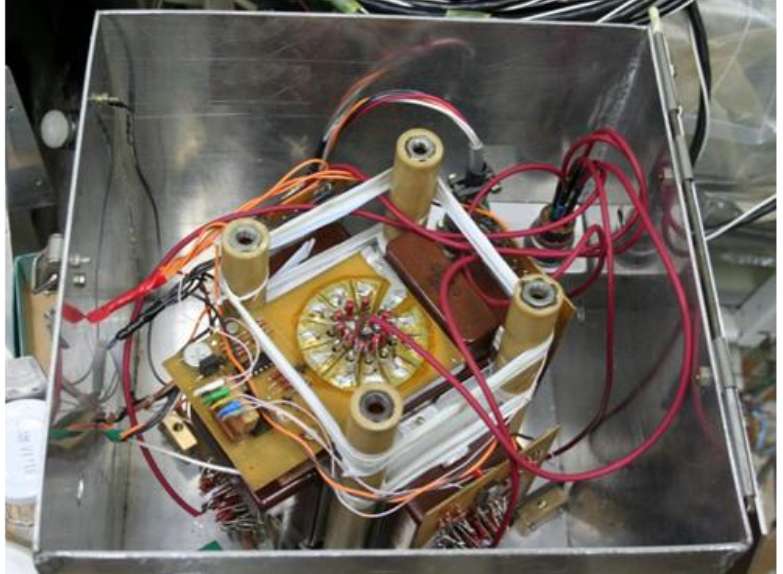

Рис. 1. Общий вид нейтронного детектора

на на рис. 1. Мёртвое время СБТ-10 составляет 40 мкс. Динамический диапазон регистрации составляет более четырёх порядков. Он определяется с нижней стороны естественным фоном радиации, дававшим обычно 100-150 отсчётов за время полураспада, а с верхней стороны - частотной характеристикой счётчиков.

Питание счётчиков +400 В подводится по кабелю из блока управления. Каждый счётчик СБТ-10 содержит 10 отдельных ячеек, осуществляющих счёт независимо друг от друга. Для реализации максимально возможной скорости счёта сигналы с каждой ячейки подаются на схему 10ИЛИ, т.е. суммарно шесть счётчиков работают на схему 60ИЛИ. С неё суммарный сигнал подаётся на мощный выходной каскад на буфере-драйвере 40107 с открытым стоком, нагруженным на кабель с волновым сопротивлением 75 Ом. Нагрузка кабеля 75 Ом размещена в блоке управления. Электронная часть блока счётчиков собрана на микросхемах серии КМОП, для их защиты от помех по питанию используются конденсаторные фильтры и защитные диоды TRANSIL. Блок управления также выполнен на микросхемах КМОП. Он включает в себя схему согласования входного сигнала, схему управления функциями измерения («ПУСК», «СТОП», «СБРОС», «ВРЕМЯ ИЗМЕР.»), шестиразрядный десятичный счётчик с индикацией, таймерхронизатор, счётчик времени измерения с индикацией. В состав блока также входит схема запуска внешним электрическим сигналом с гальванической развязкой от входного импульса на оптронах. Кроме того, блок содержит стабилизированные источники питания микросхем и счётчиков. Для защиты от помех по цепи питания 220 В используются варисторы и диоды TRANSIL. На задней стенке блока управления расположен контрольный разъём, на который выведены ключевые сигналы для проверки работы блока и комплекса в целом.

Для минимизации воздействия помех измерительный комплекс запускается спустя некоторое регулируемое время после срабатывания импульсной установки. Эта задержка устанавливается равной $0,01,0,1$ или 0,8 с с помощью двух переключателей, находящихся на плате управления в блоке управления. Режим внешнего запуска подключён постоянно и независимо от режима ручного управления. Это означает, что для запуска комплекса достаточно подать импульс любой полярности и длительностью более 50 нс на гнездо СР-50 на задней стенке блока управления. Амплитуда импульса должна быть не менее $40 \mathrm{~B}$, входное сопротивление блока по этому сигналу 10 кОм. При запуске с гальванической развязкой пусковой импульс подаётся по схеме: один конец - центральный электрод разъёма СР-50, второй конец - дополнительное гнездо. В этом случае дополнительное гнездо от корпуса отсоединяется.

Следует отметить, что такая схема измерений полностью исключает как влияние импульсных электромагнитных помех, так и непосредственное влияние рентгеновского излучения на датчики измерительной аппаратуры, что является немаловажным обстоятельством при работе на мощных импульсных генераторах. Кроме того, эксперимент с очень большим нейтронным выходом в такой схеме регистрации не пропадает. Измерение производится спустя определённое время задержки, когда в процессе распада количество отсчётов уменьшится и попадёт в динамический диапазон детектора. После чего производится пересчёт на время задержки, дающий величину нейтронного выхода. Таким образом, реальный динамический диапазон детектора увеличивается почти ещё на порядок вверх, когда уже потребуется учитывать второй канал распада ${ }^{116}$ In с временем полураспада 55 мин.

\section{ГРАДУИРОВКА ДЕТЕКТОРА}

Чувствительность детектора $\varphi$ определялась градуировкой с помощью изотопного источника нейтронного излучения, имеющего известную активность.

Число отсчётов детектора $N$ связано с количеством активированных ядер в изотопе ${ }^{116}$ In к моменту начала регистрации. Для источника с постоянной активностью $A$ (нейтр./с) число отсчётов составляет $N(t)=\Omega \varphi \int_{0}^{T} A 2^{\theta-T / \tau} d \theta\left(1-2^{-t / \tau}\right)=\Omega \varphi A \frac{\tau}{\ln 2}\left(1-2^{-T / \tau}\right)\left(1-2^{-t / \tau}\right)$, где $\Omega$ - телесный угол, кото- 
рый составляет датчик относительно источника; интеграл связан с временем накопления активности $T$ в изотопе с временем полураспада $\tau$, круглая скобка определяет часть распавшихся ядер за время регистрации $t$, начинающейся сразу после окончания облучения нейтронами. За время полураспада число отсчётов составит

$$
N_{1 / 2}=\Omega \varphi A \frac{\tau}{2 \ln 2}\left(1-2^{-T / \tau}\right)
$$

Аналогично выглядит формула для импульсного источника излучения, имеющего полный выход нейтронов $\mathfrak{R}$, время воздействия которого на детектор $T_{i}<<\tau$ :

$$
N_{1 / 2}=\Omega \varphi \frac{\Re}{2} .
$$

Для градуируемых детекторов $\Omega=\Phi_{r} /(4 \pi)$, где $\Phi_{r}-$ угловой размер ближайшей к источнику грани полиэтиленового куба замедлителя детектора.

Градуировка проведена в Институте физики высоких энергий (г. Протвино) на источнике ${ }^{252} \mathrm{Cf}$ с активностью $A=8,06 \cdot 10^{6}( \pm 8 \%)$ нейтр./с на момент проведения работы.

Процедура градуировки была следующей. Сначала измерялись фоновые отсчёты детекторов в режиме непрерывного счёта в течение 2-3 мин. Потом источник нейтронов извлекался из полиэтиленового контейнера-сферы, удалённого на $\sim 8$ м от детектора, и подносился к детектору для экспонирования на расстояние $h=8$ см от ближайшей грани полиэтиленового куба замедлителя. Через время $T=60 \mathrm{c}-$ время накопления активности источник возвращался обратно в контейнер, и одновременно включалось пересчётное устройство.

Счёт вёлся с использованием штатного блока управления в течение нескольких полупериодов распада $t=14,1$ с (время жёстко выставлено в блоке управления) с интервалами, необходимыми для записи показаний детектора с шестиразрядного индикатора. Интервалы между полупериодами регистрации фиксировались. Остановка регистрации показаний счётчика производилась после уверенного выхода показаний на фоновый уровень.

Табл. 1, 2 содержат результаты градуировки двух идентичных детекторов в указанном режиме.

Т а б л и ц а 1. Градуировка детектора № 1

\begin{tabular}{c|c|c|c|c}
\hline Время от начала регистрации, с & Отсчёт & Отсчёт без фона & Погрешность & Примечание \\
\hline 0 & 637 & $469 N_{1}(\tau)$ & 28 & \\
19 & 337 & 169 & 22 & \\
36 & 273 & 105 & 21 & \\
53 & 189 & 21 & 19 & \\
72 & 169 & 1 & 18 & Фон 168 \\
96 & 182 & 14 & 18 & \\
\hline
\end{tabular}

Т а б л и ц а 2. Градуировка детектора № 2

\begin{tabular}{c|c|c|c|c}
\hline Время от начала регистрации, с & Отсчёт & Отсчёт без фона & Погрешность & Примечание \\
\hline 0 & 637 & $453 N_{2}(\tau)$ & 28 & \\
20 & 330 & 146 & 23 & \\
40 & 232 & 48 & 20 & \\
57 & 200 & 16 & 19 & \\
74 & 192 & 8 & 20 & \\
92 & 211 & 27 & 19 & Фон 184 \\
110 & 188 & 4 & \\
\hline
\end{tabular}

Формула (1) при подстановке фактических параметров градуировки перепишется как

$$
N_{1 / 2}=\Omega \varphi A \frac{\tau}{2 \ln 2}\left(1-2^{-T / \tau}\right)=0,077 \varphi 8,06 \cdot 10^{6} \frac{14,1}{2 \cdot 0,69}(1-0,0524)=6,0 \cdot 10^{6} \varphi .
$$

По результатам измерений $N(\tau)$ получаем: чувствительность первого детектора $\varphi_{1}^{\prime}=(469 \pm 28) / 6 \cdot 10^{6}=$ $=(7,8 \pm 0,5) 10^{-5}$ нейтр. $^{-1}$; чувствительность второго детектора $\varphi_{2}^{\prime}=(453 \pm 28) / 6 \cdot 10^{6}=(7,5 \pm 0,5) 10^{-5}$ нейтр. $^{-1}$. Величина указанной погрешности соответствует статистике отсчётов.

Функция распределения нейтронов по энергии при спонтанном распаде ${ }^{252} \mathrm{Cf} \rightarrow{ }^{137} \mathrm{I}+{ }^{112} \mathrm{Rh}+3 n$ (6,17 МэВ) аппроксимируется как

$$
F(E) \sim \exp (-E / 1,25) \operatorname{sh}(2,926 E)^{1 / 2}
$$


или как

$$
f(E)=2(E /(2,9 \pi))^{1 / 2} \exp (-E / 1,427)
$$

где $E$ - энергия, МэВ, [1] (рис. 2) с максимумом $E_{\mathrm{Cf}} \sim 0,9$ МэВ, полуширина распределения превосходит 2 МэВ.

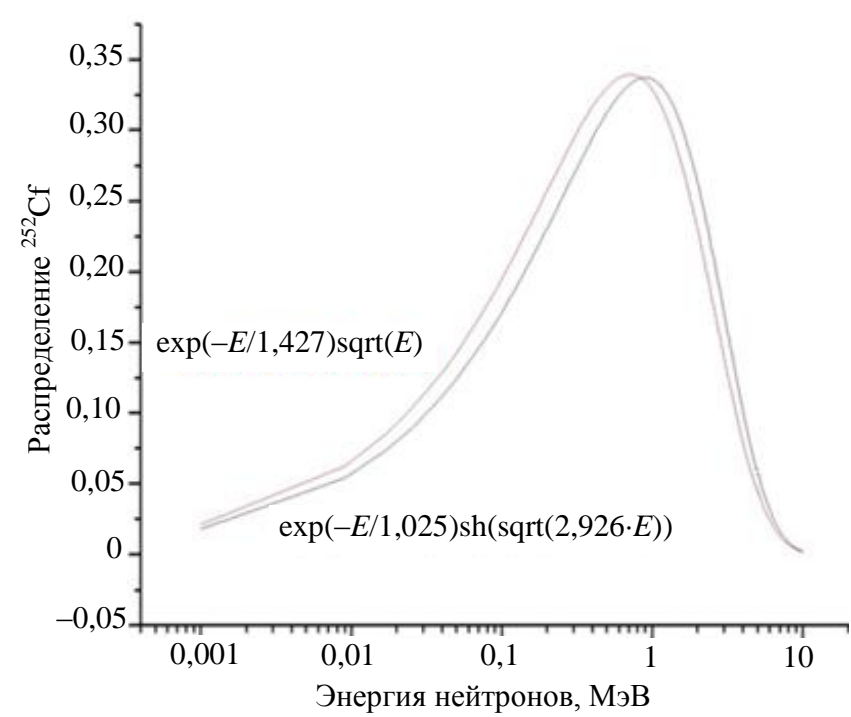

Рис. 2. Распределение нейтронов от ${ }^{252} \mathrm{Cf}$ по энергии

Представляется несущественным отличие энергии нейтронов калибровочного источника от энергии $\mathrm{D}-\mathrm{D}$-нейтронов $E_{\mathrm{D}-\mathrm{D}}=2,45$ МэВ, для измерения полного выхода которых предназначается детектор, поскольку процесс замедления для всех нейтронов ${ }^{252} \mathrm{Cf}$ до энергии 1,46 эВ резонансного поглощения в индии требует в среднем 16 столкновений с атомами водорода, которые в основном работают в полиэтилене. Для нейтронов, соответствующим краям полуширины распределения, количество столкновений для требуемого замедления отличается на \pm 1 ( $6 \%$ ). Это означает, что чувствительность детектора к $\mathrm{D}$ - D-нейтронам должна не сильно отличаться от величины, полученной при градуировке с применяемым источником. Для термализации нейтронов до комнатной температуры, при которой свободные нейтроны долго живут до распада и активация также велика, разница в количестве соударений ещё менее значительна.

\section{ЧИСЛЕННОЕ МОДЕЛИРОВАНИЕ ЗАМЕДЛЕНИЯ И ЗАХВАТА НЕЙТРОНОВ}

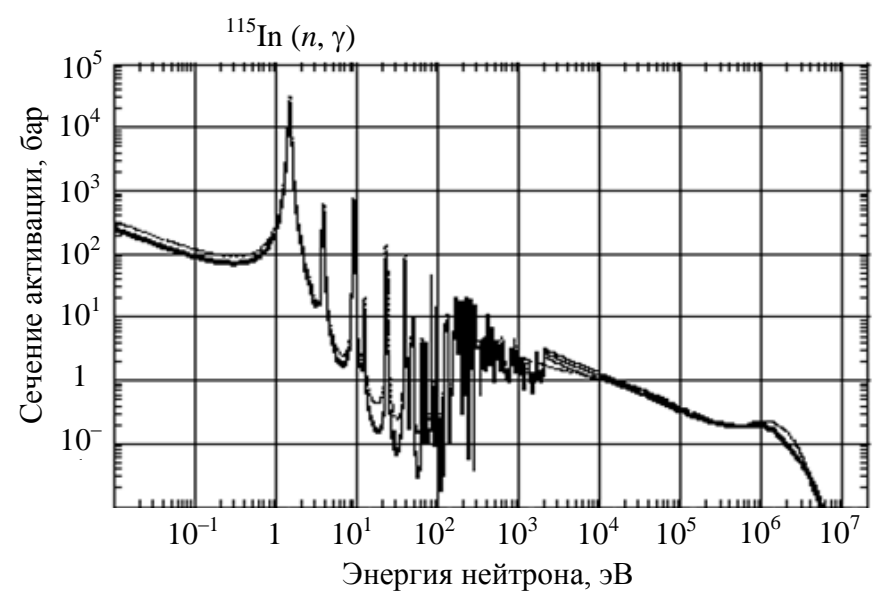

Рис. 3. Полное сечение активации индия

Кроме умозрительных заключений, проведены сравнительные расчёты для нейтронов от разных источников с помощью двух разных кодов. Считались замедление нейтронов и активация ими индиевых пластин. Полное сечение активации индия представлено на рис. 3 [2].

Расчёты по программе MCNP4c [3] проведены с двумя источниками: для монохроматического источника с $E=2,2$ МэВ и распределения в виде (3). В этих расчётах геометрия соответствовала взаимному расположению источника и детектора в процессе градуировки, а именно: источник нейтронов точечный изотропный в точке $(0,0,0)$. Полиэтиленовый куб: ребро - 9 см, центр $(0,0,12,5$ см). Результаты расчётов средней плотности потока нейтронов через лицевую, боковую и заднюю поверхность полиэтиленового куба представлены в табл. 3.

Т а б л и ц а 3 . Усреднённая по грани плотность потока нейтронов через лицевую, боковую и заднюю грань полиэтиленового куба. Нормировка на 1 нейтрон источника

\begin{tabular}{c|c|c|c}
\hline Грань & Спектр & $\begin{array}{c}\text { Усреднённая по грани плотность потока нейтронов } \\
\text { с энергией } 10^{-2}-10 \text { эВ смм }\end{array}$ & $\begin{array}{c}\text { Статистическая } \\
\text { погрешность, \% }\end{array}$ \\
\hline \multirow{2}{*}{ Лицевая } & ${ }^{252} \mathrm{Cf}(3)$, & $3,01577 \cdot 10^{-5}$ & 0,0055 \\
& $2,2 \mathrm{MэB}$ & $1,98682 \cdot 10^{-5}$ & 0,0130 \\
\multirow{2}{*}{ Задняя } & ${ }^{252} \mathrm{Cf}(3)$, & $9,36719 \cdot 10^{-6}$ & 0,0097 \\
& $2,2 \mathrm{MэB}$ & $1,04396 \cdot 10^{-5}$ & 0,0185 \\
\multirow{2}{*}{ Боковая } & ${ }^{252} \mathrm{Cf}(3)$, & $1,86635 \cdot 10^{-5}$ & 0,0069 \\
& $2,2 \mathrm{MэB}$ & $1,58781 \cdot 10^{-5}$ & 0,0155 \\
\hline
\end{tabular}


На рис. 4 представлено распределение плотности потока нейтронов $\left(\mathrm{cm}^{-2}\right)$ по боковой грани куба вдоль оси $z$ для моноэнергетических нейтронов $E=2,2$ МэВ.

Поскольку вероятность активации индия толщиной 1,5 мм медленными и тепловыми нейтронами близка к единице, то поток нейтронов через пластины практически соответствует количеству активированных ядер.

Расчёт по программе MCNP4c потока нейтронов утечки с энергией ниже 10 эВ со всех граней куба в зависимости от начальной энергии нейтронов приведён на рис. 5. Как видно на рисунке, чувствительность нашего детектора может меняться в зависимости от первичной энергии нейтронов (от 10 эВ до 14 МэВ) в диапазоне двух порядков.

Среднее количество медленных и тепловых нейтронов для двух рассчитанных источников составило $5,18 \cdot 10^{-3}$ и $6,31 \cdot 10^{-3}$ от полного числа изотропно испущенных источником нейтронов, или 0,067 и 0,082 от количества нейтронов, вошедших в лицевую грань полиэтиленового куба, соответственно. Соотношение активированных ядер индия для двух расчётных случаев оказалось равным 0,82 .

Расчёты по программе MCU-5 [4] были проведены для бесконечно удалённого монохроматического источника нейтронов с $E_{\mathrm{D}-\mathrm{D}}=2,45 \mathrm{MэB} \mathrm{и}$ распределения в виде (4), которые пускались на лицевую грань полиэтиленового куба-замедлителя нормально к его поверхности. В табл. 4 представлены расчёты активации ядер индия по энергетическим группам для источника с начальной энергией 2,45 МэB.

Т а б л и ц а 4. Количество активированных ядер индия при замедлении на один попавший в полиэтиленовый куб-замедлитель нейтрон с начальной энергией 2,45 МэВ

\begin{tabular}{|c|c|c|c|}
\hline Группы по энергии (нижняя граница), эВ & Лицевая грань & Задняя грань & Боковые грани \\
\hline $1 \cdot 10^{6}$ & $2,215 \cdot 10^{-4}$ & $8,670 \cdot 10^{-5}$ & $2,808 \cdot 10^{-4}$ \\
\hline $1 \cdot 10^{5}$ & $4,937 \cdot 10^{-5}$ & $6,751 \cdot 10^{-5}$ & $3,454 \cdot 10^{-4}$ \\
\hline $1 \cdot 10^{4}$ & $7,370 \cdot 10^{-5}$ & $5,506 \cdot 10^{-5}$ & $3,034 \cdot 10^{-4}$ \\
\hline $1 \cdot 10^{3}$ & $1,412 \cdot 10^{-4}$ & $9,144 \cdot 10^{-5}$ & $4,943 \cdot 10^{-4}$ \\
\hline $1 \cdot 10^{2}$ & $2,066 \cdot 10^{-4}$ & $1,323 \cdot 10^{-4}$ & $6,966 \cdot 10^{-4}$ \\
\hline 10 & $1,820 \cdot 10^{-4}$ & $1,186 \cdot 10^{-4}$ & $6,067 \cdot 10^{-4}$ \\
\hline 4 & $2,397 \cdot 10^{-4}$ & $1,595 \cdot 10^{-4}$ & $8,153 \cdot 10^{-4}$ \\
\hline 3 & $2,238 \cdot 10^{-4}$ & $1,552 \cdot 10^{-4}$ & $7,462 \cdot 10^{-4}$ \\
\hline 2 & $2,620 \cdot 10^{-4}$ & $1,775 \cdot 10^{-4}$ & $8,971 \cdot 10^{-4}$ \\
\hline 1 & $1,153 \cdot 10^{-3}$ & $8,000 \cdot 10^{-4}$ & $3,926 \cdot 10^{-3}$ \\
\hline $8 \cdot 10^{-1}$ & $3,308 \cdot 10^{-4}$ & $2,298 \cdot 10^{-4}$ & $1,143 \cdot 10^{-3}$ \\
\hline $6 \cdot 10^{-1}$ & $3,480 \cdot 10^{-4}$ & $2,403 \cdot 10^{-4}$ & $1,185 \cdot 10^{-3}$ \\
\hline $4 \cdot 10^{-1}$ & $4,207 \cdot 10^{-4}$ & $2,983 \cdot 10^{-4}$ & $1,437 \cdot 10^{-3}$ \\
\hline $2 \cdot 10^{-1}$ & $7,240 \cdot 10^{-4}$ & $5,202 \cdot 10^{-4}$ & $2,520 \cdot 10^{-3}$ \\
\hline 0 & $1,068 \cdot 10^{-2}$ & $8,293 \cdot 10^{-3}$ & $3,801 \cdot 10^{-2}$ \\
\hline
\end{tabular}

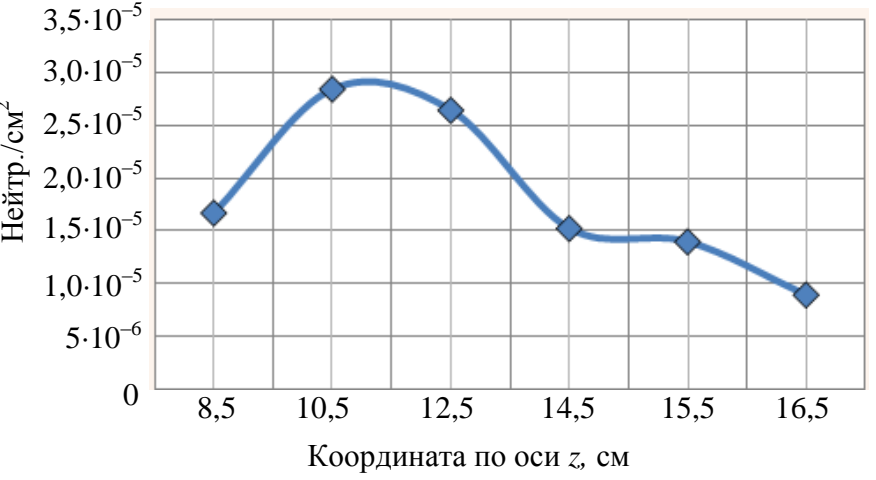

Рис. 4. Расчётное (программа MCNP4c) распределение плотности потока нейтронов по боковой грани куба вдоль оси $z$. $E=2,2$ МэВ; $x=0 ; y=4,6$

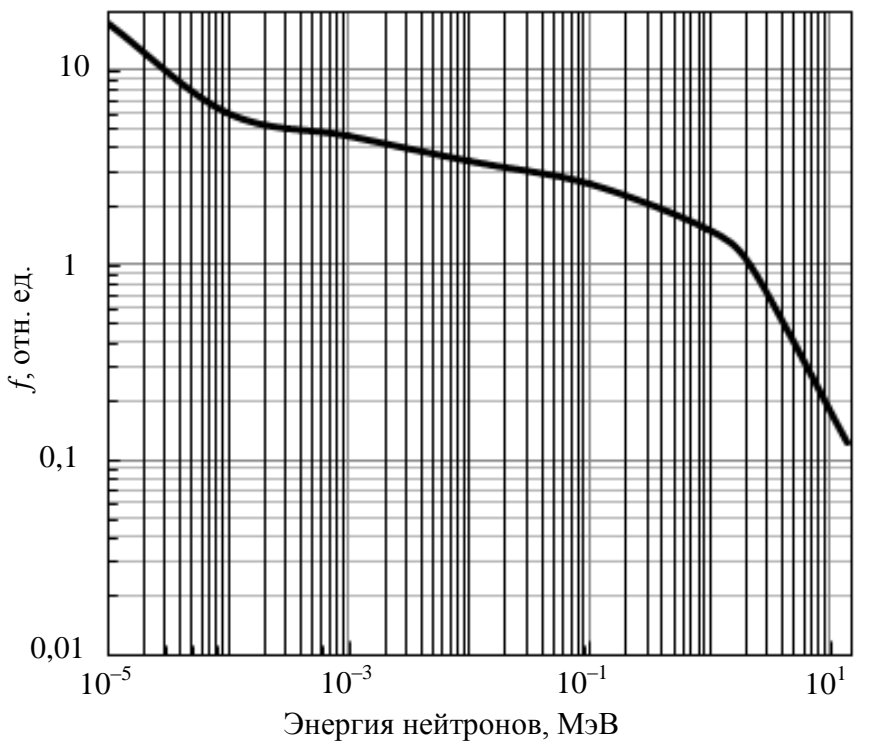

Рис. 5. Поток нейтронов утечки $f$ из куба в зависимости от начальной энергии нейтронов. Нормировка на значение при энергии нейтронов 2,2 МэВ 
Среднее количество активированных ядер индия от количества нейтронов, вошедших в лицевую грань полиэтиленового куба, составило 0,08 и 0,102 для двух источников соответственно. Соотношение активированных ядер индия для этих случаев оказалось равным 0,784 .

Полученные отношения для двух вычислительных программ отличаются на 4\%, что, по-видимому, связано с включением в расчёт процесса активации индия. Таким образом, в величину чувствительности следует ещё ввести расчётный поправочный коэффициент $k_{E} \cong 0,8$, связанный с различием энергии нейтронов калибровочного источника и нейтронов в D-D-реакции.

Поскольку приближение бесконечно удалённого источника справедливо в случае реального эксперимента, где детектор располагается на удалении $\geq 1,2$ м от источника излучения, но не соответствует геометрии, в которой проводилась градуировка, то по программе MCU-5 ещё был сделан сравнительный расчёт для точечного источника ${ }^{252} \mathrm{Cf}$, расположенного на 12,5 см от центра куба, т.е. на $h=8 \mathrm{~cm}$.

Сделаем необходимое пояснение последнего расчёта. Обозначим $r$ половину ребра куба, т.е. $r=4,5$ см. Для того чтобы не моделировать нейтроны, летящие в пустоту, а таких большинство в силу изотропности источника, их направления были равномерно распределены по шаровому сегменту, определённому неравенством $0 \leq \theta \leq \theta_{m}$, где $\theta_{m}=\operatorname{arctg}\left(2^{1 / 2} r / h\right)$. Ясно, что площадь этой «шапочки» есть $S_{m}=2 \pi\left(1-\cos \left(\theta_{m}\right)\right)$.

В расчётах с мононаправленным распределением нейтронов источник был выбран так, что все нейтроны попадают в куб. При сопоставлении с точечным источником нужно ещё нормировать лишь на те частицы, которые попадают в куб, а из «шапочки» попадают не все, а лишь те, которые лежат на сфере в проекции лицевой грани куба - это криволинейный четырёхугольник. Его площадь, очевидно, есть

$$
S_{r}=2 \pi-8 \int_{0}^{\pi / 4} \cos (t) d t / \sqrt{\cos ^{2}(t)+(r / h)^{2}},
$$

этот интеграл нетрудно взять в явном виде

$$
I=\int_{0}^{\pi / 4} \cos (t) d t / \sqrt{\cos ^{2}(t)+(r / h)^{2}}=\arcsin (\sqrt{2} /(2 a)),
$$

где число $a$ определено соотношением $a^{2}=1+r^{2} / h^{2}$. Так что $a=1,1473 ; I=0,66403 ; S_{r}=0,97093$; $S_{m}=1,36606 ; S_{r} / S_{m}=0,71075$.

Выданное программой MCU-5 число поглощения на индии 0,0499 следует разделить на отношение площадей $S_{r} / S_{m}$. В результате получится оценка 0,0702 (в расчёте по программе MCNP4c получилось 0,067, что даёт отличие в 5\%), в мононаправленном источнике с калифорнием она была 0,102.

Таким образом, в величину чувствительности следует ещё ввести расчётный поправочный коэффициент для использованной при градуировке геометрии $k_{G} \cong 0,102 / 0,0702 \cong 1,46$.

Обычно в дозиметрии нейтронов для точечного детектора и точечного источника принимают в качестве чувствительности отклик детектора на единичную плотность потока нейтронов в месте расположения детектора. Когда размер детектора сравним с расстоянием $R$ при градуировке, вводят поправку $\rho$, которая при $R>6 r$ (где $2 r$ - характерный геометрический диаметр полиэтиленового замедлителя) уже принимается равной единице. Обычно значение поправки $\rho$ определяется экспериментально. В нашем случае при недостаточной для такой градуировки активности источника был выполнен расчёт для точечного источника нейтронов ${ }^{252} \mathrm{Cf}$ по программе MCNP4C. На рис. 6 показа-

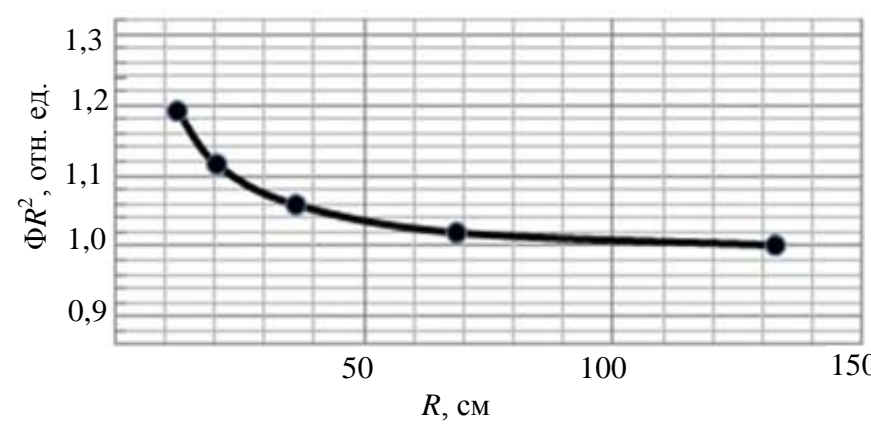

Рис. 6. Зависимость поправки $\rho=\Phi R^{2}$ от расстояния $R$ на зависимость произведения $\Phi \cdot R^{2}$ от $R$, где $\Phi-$ поток нейтронов утечки с энергией $10^{-2}-10$ эВ из полиэтиленового куба, $R$ - расстояние от точечного источника до центра куба. Нормировка на единицу приведена для расстояния 128 см. Поправка для $R=12,5$ см составила $\rho=1,19$, значение которой отличается от посчитанного программой MCU-5 коэффициента $k_{G}=1,46$, по-видимому, в связи с включением в расчёт процесса активации индия. 
Значение чувствительности двух детекторов в формуле (1) для $E_{\mathrm{D}-\mathrm{D}}=2,45$ МэВ после коррекции на энергетическое распределение нейтронов в калибровочном источнике и на геометрию измерений составит:

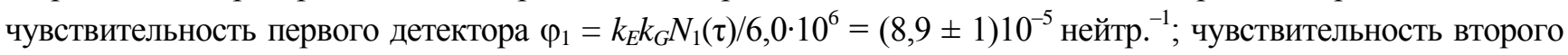

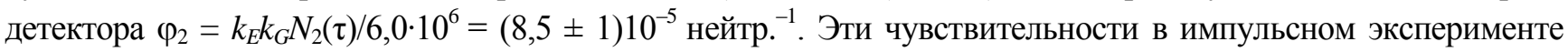
подставляются для определения нейтронного выхода в формулу (2).

\section{ЗАМЕЧАНИЕ О КАЛИБРОВОЧНОМ ИСТОЧНИКЕ}

Для градуировки нейтронных детекторов использовался радиоактивный источник на основе изотопа ${ }^{252} \mathrm{Cf}$. Гарантийный срок использования источника составляет по паспорту 15 лет. К моменту испытаний возраст источника составил $T_{\mathrm{Cf}}=24,43$ года, вследствие чего произошло значительное изменение его изотопного состава.

Как известно (табл. 5), ${ }^{252} \mathrm{Cf}$ даёт мгновенные нейтроны в результате спонтанного распада ${ }^{252} \mathrm{Cf} \rightarrow{ }^{137} \mathrm{I}+{ }^{112} \mathrm{Rh}+3 n$. Если проследить ветви дальнейшего распада этих осколков, то в результате серии $\beta$-распадов получаются стабильные изотопы: ${ }^{137} \mathrm{I}_{53} 94 \% \rightarrow\left(\beta^{-} ; 22 \mathrm{c}\right){ }^{137} \mathrm{Xe}_{54} \rightarrow\left(\beta^{-} ; 9,3 \mathrm{M}\right){ }^{137} \mathrm{Cs}_{55} \rightarrow\left(\beta^{-}\right.$; 30 л) ${ }^{137} \mathrm{Ba}_{56} \rightarrow(\gamma)$ стаб., или ${ }^{137} \mathrm{I}_{53} 6 \% \rightarrow\left(\beta^{-} n ; 22 \mathrm{c}\right){ }^{136} \mathrm{Xe}_{54}$ стаб.

Излучение дополнительных нейтронов имеет короткий полупериод распада и явно включается в первоначальную нейтронную активность калифорния.

Второй вид распада ${ }^{252} \mathrm{Cf}-\alpha$-распад не так безобиден. В результате получается ядро ${ }^{248} \mathrm{Cm}$, которое долго живёт и даёт нейтроны в спонтанном распаде (см. табл. 5) [5].

Т а б л и ц а 5. Спонтанный распад калифорния и нарабатываемого им кюрия

\begin{tabular}{|c|c|c|c|c|}
\hline Изотоп & Время полураспада & Вид распада & Излучение и его энергия, МэВ & $\begin{array}{c}\text { Количество и средняя } \\
\text { энергия нейтронов, МэВ }\end{array}$ \\
\hline${ }^{252} \mathrm{Cf}$ & 2,645 года & $\begin{array}{l}\alpha 96,91 \% \\
\text { SF } 3,09 \%\end{array}$ & $\begin{array}{l}\alpha 6,118(84 \%) \\
6,076(15,8 \%)\end{array}$ & $3,7675,1,61$ \\
\hline${ }^{248} \mathrm{Cm}$ & 348000 лет & $\begin{array}{l}\alpha 91,61 \% \\
\text { SF } 8,39 \%\end{array}$ & $\begin{array}{l}\alpha 5,078(82 \%) \\
5,034(18 \%)\end{array}$ & $3,157,1,59$ \\
\hline
\end{tabular}

Оценим отношение интенсивности излучения нейтронов от первичного калифорния к интенсивности излучения нейтронов от кюрия. Интенсивность излучения нейтронов калифорнием равна

$$
N_{\mathrm{Cf}}=A \cdot 2^{-T / \tau_{\mathrm{Cr}}} .
$$

Для интенсивности излучения нейтронов кюрием

$$
N_{\mathrm{Cm}}=A \frac{96,9 \%}{3,1 \%} \frac{\tau_{\mathrm{Cf}}}{\tau_{\mathrm{Cm}}} \frac{n_{\mathrm{Cm}}}{n_{\mathrm{Cf}}}\left(1-2^{-T / \tau_{\mathrm{Cf}}}\right) 8,39 \% .
$$

В этой формуле $n_{\mathrm{Cf}}$ и $n_{\mathrm{Cm}}-$ среднее число нейтронов, испускаемых в одном акте ядром, соответствующим нижнему индексу, проценты вероятности спонтанного распада и $\alpha$-распада взяты из табл. 5 :

$$
K=\frac{N_{\mathrm{Cf}}}{N_{\mathrm{Cm}}}=\frac{3,1 \% \cdot 2^{-T / \tau_{\mathrm{Cf}}}}{96,9 \% \cdot 8,39 \% \cdot\left(1-2^{-T / \tau_{\mathrm{Cf}}}\right)} \frac{\tau_{\mathrm{Cm}}}{\tau_{\mathrm{Cf}}} \frac{n_{\mathrm{Cf}}}{n_{\mathrm{Cm}}}=\frac{3,1 \% \cdot 2^{-24,43 / 2,645}}{96,9 \% \cdot 8,39 \% \cdot\left(1-2^{-24,43 / 2,645}\right)} \frac{348000}{2,645} \frac{3,7675}{3,157}=99,4 .
$$

Получается, что наработанный к моменту градуировки кюрий даёт ещё только $1 \%$ быстрых нейтронов от интенсивности калифорния. Энергетическое распределение спонтанных нейтронов кюрия практически такое же, как и у калифорния. Таким образом, активность источника всё ещё определяется изотопом ${ }^{252} \mathrm{Cf}$.

\section{ВЫВОДЫ}

Итак, разработан и проградуирован детектор D-D-нейтронов на основе активации ядер индия для импульсной $\left(10^{-8}\right.$ c) реакции синтеза, осуществляемой в экспериментах на мощных генераторах тока. Детектор имеет эффективный динамический диапазон регистрации более пяти десятичных порядков. Он осуществляет пересчёт активированных ядер после срабатывания генератора, что исключает воздействие электромагнитных помех на регистрацию.

Формулу определения полного выхода нейтронов $\mathfrak{R}$ для импульсного источника излучения удобно представить в виде

$$
\mathfrak{R}=N_{1 / 2} R^{2} \xi,
$$


где $N_{1 / 2}$ - число отсчётов детектора за первый полупериод распада индия $(14,1 \mathrm{c}) ; R$ - удаление центра детектора от источника нейтронов, см; $\xi$ - пересчётный коэффициент, равный $(1,43 \pm 0,2) 10^{3}$ и $(1,5 \pm 0,2) 10^{3}$ для первого и второго детектора соответственно.

В указанную погрешность включены статистика отсчётов во время градуировки, неопределённость в активности источника излучения и разница в расчётах по двум вычислительным программам.

Желательно располагать детектор от источника на расстоянии, превосходящем 1 м, чтобы не вводить поправочные коэффициенты на «неточечность» детектора.

Следует обратить внимание на последнюю строку в табл. 4, которая показывает определяющее значение тепловых нейтронов в активации индия. В случае расположения детекторов вблизи стен и конструкций экспериментальных стендов настоятельно рекомендуется сделать для них индиевую или кадмиевую защиту от тепловых нейтронов.

Работа поддержана грантом РФФИ № 11-02-00492-а, грантом Президента РФ НШ-4463.2012.2 и ГКП1102.

\section{REFERENCES}

1. Tables of physical magnitudes. Handbook. Edited by I.K. Kikoin. - Moscow: Atomizdat, 1976 (in Russian).

2. http://www.iaea.org/inis/collection/NCLCollectionStore/_Public/37/001/37001610.pdf.

3. MCNP - a General Monte Carlo N-Particle Transport Code, Version 4C. Ed. by J.F. Briesmeister. LA13709-M, April 2000.

4. Gomin E.A. Status of MCU. - VANT. Ser. Fizika yadernykh reaktorov (Problems of Atomic Science and Technology. Ser. Physics of nuclear reactors), 2011 (in press) (in Russian).

5. http://radchem.nevada.edu/classes/rdch710/files/californium.pdf and .../curium.pdf.

\section{AUTHORS}

Dan'ko S.A. NRC “Kurchatov Institute”, pl. Kurchatova 1, 123182 Moscow, Russia; danko@ dap.rncki.ru

Kingsep S.S. NRC “Kurchatov Institute”, pl. Kurchatova 1, 123182 Moscow, Russia; sergsk49@yandex.ru

Tel'kovskaya O.V. NRC “Kurchatov Institute”, pl. Kurchatova 1, 123182 Moscow, Russia; gur.m@mail.ru

Alekseev A.G. Institute for High Energy Physics, ul. Pobedy 1, 142280 Protvino, Moscow region, Russia; Alexander.Alexeev@ihep.ru

Britvich G.I. Institute for High Energy Physics, ul. Pobedy 1, 142280 Protvino, Moscow region, Russia; britvich@ihep.ru, gur.m@mail.ru 